\title{
Consensus for Multiagent Systems with Nonlinear Dynamics and Time Delays Using a Two-Hop Relay Adaptive Method
}

\author{
Qian Cao, ${ }^{1}$ Y. D. Song, ${ }^{1,2}$ Lei Wang, ${ }^{1,2}$ and Feng Yang ${ }^{1}$ \\ ${ }^{1}$ School of Energy Science and Engineering, University of Electronic Science and Technology of China, Chengdu 611731, China \\ ${ }^{2}$ School of Automation, Chongqing University, Chongqing 400044, China \\ Correspondence should be addressed to Lei Wang; leiwang08@cqu.edu.cn
}

Received 1 April 2014; Accepted 6 May 2014; Published 21 May 2014

Academic Editor: Shen Yin

Copyright (c) 2014 Qian Cao et al. This is an open access article distributed under the Creative Commons Attribution License, which permits unrestricted use, distribution, and reproduction in any medium, provided the original work is properly cited.

\begin{abstract}
This paper investigates the consensus problem for multiagent systems with nonlinear dynamics and time delays. A distributed adaptive consensus protocol is proposed in which the time delays are explicitly included in the adaptive algorithm. It is shown that the resultant closed loop system involves doubly larger time delays, making the stability analysis nontrivial. Stability condition on maximum tolerable time delay is established and controlled by the proposed two-hop adaptive algorithm. The explicit expression of the delay margin is derived and analyzed in the frequency domain. Both the agent state errors and the estimation parameter errors converge to zero. A simulation example is illustrated to verify the theory results.
\end{abstract}

\section{Introduction}

As one of the most typical and important problems of multiagent systems, consensus problem has received increasing attention in various fields, such as economy, electrical engineering, and physics. Many literatures have addressed the consensus problem for multiagent systems by using matrix theory, the frequency-domain analysis method, Lyapunovbased approach, and so forth. Consensus problem for firstorder multiagent systems was firstly investigated. OlfatiSaber and Murray [1] investigated consensus problems with directed interconnection graphs or time delays by a Lyapunov-based approach. Blondel et al. [2] investigated state consensus problems for discrete-time multiagent systems with changing communication graphs that may not have spanning trees. Then Lasseter [3] extended the consensus problem into double integrated systems in investigation for decentralized stabilization of vehicle formations. Ren and Atkins [4] proposed a second-order protocol and provided sufficient conditions for networks with fixed and switching topologies. Yu et al. [5] studied the second-order consensus in multiagent systems using sampled position and velocity data. Yang et al. [6] studied the consensus of second-order multiagent dynamical systems with exogenous disturbances using a pinning control strategy.

Knowing that time delays are ubiquitous and are a key factor influencing the stability of the multiagent systems, many researchers have carried out studies on consensus problem with time delays. Yu et al. [7] obtained a necessary and sufficient condition for second-order consensus in networked multiagent systems with transmission delays. Lin et al. [8] studied the consensus problem for second-order systems with nonuniform time delays using a frequency domain approach and an upper bound of the maximum of the time-delays was given. Tian and Liu [9] studied the consensus problem with both diverse input and communication time delays and pointed out that only the input time delays have influence on system stability while communication delays do not. Besides the above mentioned works, extensive achievement has been made on consensus with time delays in numerous literatures [10-14].

It is also noticed that consensus problem with nonlinear dynamics has become an interesting topic recently as nonlinearities are inevitable in practical engineering. Multiple agent systems with nonlinear dynamics have been extensively studied during recent years [15-21]. Yu et al. [22] studied 
a nonlinear second-order consensus with directed topologies and derived some sufficient conditions for reaching a consensus. Wen et al. [23] investigated the consensus of nonlinear systems with intrinsic delays and intermittent communications. Yu and Xia [19] studied the consensus problem for leader-following systems by parameterizations of unknown nonlinear dynamics of agents.

Although plenty of researches on consensus problem have been carried out in respect of nonlinear dynamics, very few have at the same time addressed the time delays that are also crucial and unavoidable in the systems. Motivated by this fact, in this work, we investigate the consensus problem for multiagent systems with both nonlinear dynamics and time delays concurrently. It turns out that the underlying problem becomes quite challenging when both factors are addressed simultaneously. When the system is ideally with no time delay, adaptive method is an effective tool in control of nonlinear system. However, when applied under time delay situations, adaptive control involves more time delays in distributed systems. In order to estimate the unknown parameters, information from the agent itself and the neighbors may be acquired within multiple times during a single adaptive control period. Each time the agents obtain information from the system, the time delays accumulate once. This accumulation of time delays may have an influence on the system stability, yet, to our best knowledge, no study has been carried out to address this issue. Motivated by this concern, we studied the consensus problem for multiagent systems with both nonlinear dynamics and time delays in this work using adaptive protocols so that the agent states reach a common value and the estimation parameter errors converge to zero as well. The contribution of this work lies in threefold. First, a two-hop adaptive consensus scheme is proposed for multiagent systems with nonlinear dynamics and time delays. Second, by using a frequency domain method, a necessary condition of the maximum tolerated time delay is given for system consensus using the proposed adaptive control method. Moreover, the explicit result of the maximum time delay is derived by a frequency sweeping method. Third, by extending the order of the system, the convergences of not only the agent states but also the estimation parameter errors can both be guaranteed.

The rest of the paper is organized as follows. First, some preliminaries of graph theory and some lemmas are given and then an extended-order model of the nonlinear system with time delays is established Section 2. The frequency domain analysis for consensus with accumulated time delays is studied and a necessary condition of the upper bound of the maximum tolerated time delay for system consensus is given in Section 3. An example of a 6-node multiagent system with nonlinear dynamics and time delays is illustrated in Section 4. Finally, conclusion remarks are drawn in Section 5.

\section{Preliminaries and the Model Formation}

The information exchanging between agents is generally considered as a graph. Consider an undirected weighted graph $G=\{V, E, A\}$ with $n$ nodes, where $V=\left\{v_{i} \mid i \in \Gamma\right\}$ is the set of nodes, with each node representing an agent, and $E \subseteq V \times V$ is the set of edges, with each edge representing communication line between two agents. If $\left(v_{j}, v_{i}\right) \in E$, then node $v_{j}$ is a neighbor of node $v_{i}$. The set of neighbors of node $v_{i}$ is denoted by $N_{i}=\left\{v_{j} \mid\left(v_{j}, v_{i}\right) \in E\right\} . A=\left[a_{i j}\right] \in \mathbb{R}^{N \times N}$ is the associated adjacency matrix, which is defined such that if $\left(v_{j}, v_{i}\right) \in E$, then $a_{i j}>0$; otherwise, $a_{i j}=0$. The degree matrix $D \in \mathbb{R}^{N \times N}$ is a diagonal matrix with $i$ th element being $\operatorname{deg}\left(v_{i}\right)=\sum_{j \in N_{i}} a_{i j}$. The Laplacian matrix is defined as $L=D-A$. If there is at least one path from any two distinct nodes in the graph, this graph is said to be strongly connected. A strongly connected graph contains a spanning tree with each node as the root node.

Lemma 1 (see [24]). The Laplacian matrix L has a simple eigenvalue zero, and all the other eigenvalues have positive real parts if and only if the undirected network has a spanning tree. In other words, the eigenvalues of L satisfy $0=\lambda_{1}(L)<\lambda_{2}(L) \leq$ $\cdots \leq \lambda_{n}(L)$. Furthermore, $L \mathbf{1}_{n}=0$ holds, where $\mathbf{1}_{n}$ represents a column vector with all ones.

Consider a system which consisted of $n$ second order multiagents. The dynamics of each agent is given as follows:

$$
\dot{x}_{i}(t)=f_{i}\left(x_{i}(t), t\right)+u_{i}(t) \quad i \in \Gamma,
$$

where $x_{i}(t) \in \mathbb{R}$ and $u_{i}(t) \in \mathbb{R}$ are the position and the control input of agent $i . f_{i}\left(x_{i}(t), t\right)$ represents the inherent nonlinear dynamics of agent $i$, which can be parameterized as $f_{i}\left(x_{i}(t), t\right)=\phi_{i}^{T}\left(x_{i}(t), t\right) \theta_{i}$.

Assumption 2. The Laplace transform of the nonlinear part $f_{i}\left(x_{i}(t), t\right)$ exists and is convergence as $F_{i}(s)=$ $\int_{-\infty}^{+\infty}\left|f_{i}\left(x_{i}(t), t\right)\right| e^{-\sigma t} d t<\Delta<+\infty$.

Definition 3. The multiagent system (1) is said to achieve second-order consensus, if, for any initial conditions and for all $i, j$,

$$
\lim _{t \rightarrow \infty} x_{i}(t)=\lim _{t \rightarrow \infty} x_{j}(t)
$$

Denote that $x=\left[x_{i}\right]^{T} \in \mathbb{R}^{N}$ is the global position states of the multiagent system. The global position error is $\bar{x}(t)=$ $x(t)-(1 / n) \sum_{i=1}^{n} x_{i}(0) \mathbf{1}_{n}$. Here, we introduce the local error $e_{i}(t) \in \mathbb{R}$ as follows:

$$
e_{i}(t)=\sum_{j \in N_{i}} a_{i j}\left[x_{i}(t-\tau(t))-x_{j}(t-\tau(t))\right]
$$

where $\tau(t)$ is the input delays. Or the vector forms as

$$
e(t)=L \bar{x}(t-\tau)
$$

where $e=\left[e_{i}\right]^{T} \in \mathbb{R}^{N}$.

In most practical systems, time delays are inevitably involved due to information transmission, signal sensing, processing and calculating, and so forth. As we know, the communication delays between two connected agents have no effect on the stability of the system. Therefore, we only 
investigate the situation with input delays caused by the actuation, monitoring and sensing, and so forth. In the following, we propose a consensus protocol and the corresponding adaptive law for systems with time delays:

$$
\begin{gathered}
u_{i}(t)=-c e_{i}(t)-\hat{\theta}_{i} \phi_{i}(t), \\
\dot{\hat{\theta}}_{i}(t)=-p \phi_{i}(t) \sum_{j \in N_{i}} a_{i j}\left[e_{i}(t-\tau)-e_{j}(t-\tau)\right],
\end{gathered}
$$

where $c>0, p>0$.

Remark 4. Note that not only the protocol (5) but also the adaptive law (6) utilizes the delayed information only. The proposed adaptive scheme applies the concept of twohop relay technique from the communication area, which involves accumulated time delays in one control period [25]. Generally speaking, each time the agent gets the system information, that is, the variable vectors (here refer to $\bar{x}$ and $e$ ) which are multiplied by $L$, one hop of time delay is involved in the system. Therefore, the whole control procedure involves two scales of time delays and a two-hop communication. Next, we do some preparation work for the consensus problem using the proposed method.

The protocol and the adaptive law in (5) and (6) can be written in matrix form as

$$
\begin{aligned}
& u(t)=-c e(t)-\Phi(t) \widehat{\Theta}(t), \\
& \dot{\widehat{\Theta}}(t)=p_{1} \Phi(t) L e(t-\tau),
\end{aligned}
$$

where $\dot{\widehat{\Theta}}(t)=\left[\widehat{\theta}_{i}(t)\right]^{T} \in \mathbb{R}^{n}$ and $\Phi(t)=\operatorname{diag}\left\{\phi_{i}(t)\right\} \in \mathbb{R}^{n \times n}$.

Denote $\bar{\Theta}(t)=\widehat{\Theta}(t)-\Theta(t), \delta(t)=\left[\bar{x}^{T}(t), \bar{\Theta}^{T}(t)\right]^{T}$. Then we have $\dot{\bar{\Theta}}(t)=\dot{\widehat{\Theta}}(t)=\Phi_{i}(t) L^{2} \bar{x}(t-2 \tau)$.

From (1), (4), (7), and (8), we rewrite the system dynamics as

$$
\begin{gathered}
\dot{\bar{x}}(t)=-c L \bar{x}(t-\tau)-\Phi(t) \dot{\bar{\Theta}}(t), \\
\dot{\bar{\Theta}}(t)=p L^{2} \bar{x}(t-2 \tau), \\
\dot{\delta}(t)=A \delta(t)+B \delta(t-\tau)+C \delta(t-2 \tau),
\end{gathered}
$$

where $A=\left(\begin{array}{cc}0 & -\Phi \\ 0 & 0\end{array}\right), B=\left(\begin{array}{cc}-c L & 0 \\ 0 & 0\end{array}\right)$, and $C=\left(\begin{array}{cc}0 & 0 \\ p \Phi L^{2} & 0\end{array}\right) \in \mathbb{R}^{2 n \times 2 n}$.

Lemma 5. Let $A, B$, and $C$ be defined as in (10). Then $A+B+$ $C$ has a simple zero eigenvalue, while all the other eigenvalues have negative real parts. Furthermore, $y=\varepsilon\left[\mathbf{1}_{n}^{T}, 0\right]^{T} \in \mathbb{R}^{2 n}$ is an eigenvector of the matrix $A+B+C$, where $\varepsilon \in \mathbb{R}$.

Proof. $A+B+C=\left(\begin{array}{cc}-c L & -\Phi \\ p \Phi L^{2} & 0\end{array}\right)$. It is easy to calculate its eigenvalue as

$$
\gamma_{i}=\frac{-c \lambda_{i} \pm \sqrt{c^{2} \lambda_{i}^{2}-4 p \lambda_{i}^{2} \rho_{i}^{2}}}{2} \leq 0
$$

where $\lambda_{i}, \rho_{i}$ represent the $i$ th eigenvalue of matrix $L$ and $\Phi$, respectively. According to Lemma 1 , it is clear that $A+$ $B+C$ has a simple zero eigenvalue, and $(A+B+C) y=$ $\varepsilon\left(\begin{array}{cc}-c L & -\Phi \\ p \Phi L^{2} & 0\end{array}\right)\left(\begin{array}{c}\mathbf{1}_{n}^{T} \\ 0\end{array}\right)=0$ holds.

Remark 6. The adaptive algorithm is employed to estimate the nonlinear part of the system in the proposed protocol. However, in order to calculate the estimate parameters, the system needs a two-hop communication to enlarge the information from the neighborhood and thus to implement the algorithm. In the first round, each agent obtains the local and neighboring information to calculate the global position error $e$, as is indicated in (3) and (4). Then, in the subsequence round, the agent gets the local and neighboring position errors to compute the estimation parameters $\widehat{\Theta}$, as is indicated in (6) and (8). This two-hop relay does not cause a problem in an ideal system with no time delay. However, in a practical system, time delays are inevitable and may influence the stability of the system, especially when the system is controlled within two hops, which involves two periods of time delays. To address this problem, we extend the system orders by including the estimation parameter $\widehat{\Theta}$ as a state value, as can be seen in (9). In the next section, we will analyze the stability with two time delays in frequency domain.

\section{Main Results}

In this section, by applying a frequency domain method, we analyze the maximum time delay which can be tolerant by the system to reach a consensus. Before moving on, the following lemma is given in preparation for the main results in Theorems 8 and 10 .

Lemma 7 (see [8]). For $\omega \in(0,+\infty), \alpha>0, h(\omega)=$ $(1 / \omega) \arctan (\alpha \omega)$ is decreasing.

Theorem 8. Consider a multiagent system with nonlinear $d y$ namics and time delays. The adaptive consensus protocols (7) and (8) solve the consensus problem only when the largest time delay satisfies

$$
\tau^{*}<\frac{1}{\omega_{n}} \arctan \left(\frac{\omega_{n} c}{p \lambda_{n} M^{2}}\right),
$$

where $\omega_{n}=\sqrt{\left(c^{2} \lambda_{n}^{2}+\sqrt{c^{4} \lambda_{n}^{4}+4 p^{2} \lambda_{n}^{4} M^{4}}\right) / 2} . \lambda_{n}$ is the largest eigenvalue of matrix L. $M$ is the largest absolute value of $\mu_{i}$.

Proof. Consider the system (10) in frequency domain. After the Laplace transform, we have

$$
\operatorname{det}\left(s I_{2 n}-A-B e^{-\tau s}-C e^{-2 \tau s}\right)=0
$$

or, more specifically,

$$
\begin{gathered}
\left|\begin{array}{cc}
s I_{n}+c L e^{-\tau s} & \Phi \\
-p \Phi L^{2} e^{-2 \tau s} & s I_{n}
\end{array}\right|=0, \\
s^{2}+s c \lambda_{i} e^{-\tau s}+p \lambda_{i}^{2} \mu_{i}^{2} e^{-2 \tau s}=0,
\end{gathered}
$$


where $\lambda_{i}, \mu_{i}$ represent the $i$ th eigenvalue of matrix $L$ and $\Phi(s)$, respectively.

For system (15) with time delays, the largest delay $\tau^{*}$ must be smaller than the smallest $\tau$ which satisfies (15). Otherwise, the system is not able to maintain stability.

According to the translation characteristic of Laplace transformation, we have

$$
L\left[\lambda_{i}^{2} \mu_{i}^{2} e^{-2 \tau s}\right]=J(s+\tau)
$$

where $L[\cdot]$ represents the Laplace transform. $J(s)$ is the Laplace transform of function $\lambda_{i}^{2} \mu_{i}^{2} e^{-\tau s}$; thus $J(s)=$ $L\left[\lambda_{i}^{2} \mu_{i}^{2} e^{-\tau s}\right]$. Apparently, from (16), we know that $\tau^{*}$ is smaller than its counterpart of the following equation:

$$
s^{2}+s c \lambda_{i} e^{-\tau s}+p \lambda_{i}^{2} \mu_{i}^{2} e^{-\tau s}=0 .
$$

In the following, we calculate the smallest $\tau$ that satisfies (17) thus to give an upper bound of $\tau^{*}$.

Let $s=j \omega_{i} \neq 0$. From Euler formula $e^{-\tau j \omega}=\cos (\omega \tau)-$ $j \sin (\omega \tau)$, (17) yields

$$
\omega_{i}^{2}+\left(j \omega_{i} c \lambda_{i}+\lambda_{i}^{2} \mu_{i}^{2}\right)\left[\cos \left(\omega_{i} \tau\right)-j \sin \left(\omega_{j} \tau\right)\right]=0 .
$$

By separating the real part and the imaginary part of (18), we have

$$
\begin{gathered}
\omega_{i} c \lambda_{i} \cos \left(\omega_{i} \tau\right)-p \lambda_{i}^{2} \mu_{i}^{2} \sin \left(\omega_{i} \tau\right)=0, \\
\omega_{i}^{2}+\omega_{i} c \lambda_{i} \sin \left(\omega_{i} \tau\right)+p \lambda_{i}^{2} \mu_{i}^{2} \cos \left(\omega_{i} \tau\right)=0 .
\end{gathered}
$$

Equation (20) multiplied by $\sin \left(\omega_{i} \tau\right)$ subtracts (19) multiplied by $\cos \left(\omega_{i} \tau\right)$ :

$$
\omega_{i}^{2} \sin \left(\omega_{i} \tau\right)+\omega_{i} c \lambda_{i}=0 .
$$

Similarly, (20) multiplied by $\cos \left(\omega_{i} \tau\right)$ subtracts (19) multiplied by $\sin \left(\omega_{i} \tau\right)$ :

$$
\omega_{i}^{2} \cos \left(\omega_{i} \tau\right)+p \lambda_{i}^{2} \mu_{i}^{2}=0 .
$$

By simple calculation of (21) and (22), we have

$$
\begin{gathered}
\tau=\frac{1}{\omega_{i}} \arctan \left(\frac{\omega_{i} c}{p \lambda_{i} \mu_{i}^{2}}\right), \\
\omega_{i}=\sqrt{\frac{c^{2} \lambda_{i}^{2}+\sqrt{c^{4} \lambda_{i}^{4}+4 p^{2} \lambda_{i}^{4} \mu_{i}^{4}}}{2} .}
\end{gathered}
$$

From Lemma 7, we know that when $\omega_{i}$ is the largest, $h\left(\omega_{i}\right)$ obtains the smallest value. Note from (23) that one has

$$
\tilde{\tau} \leq h\left(\omega_{n}\right)=\frac{1}{\omega_{n}} \arctan \left(\frac{\omega_{n} c}{p \lambda_{n} M^{2}}\right),
$$

where $\omega_{n}=\sqrt{\left(c^{2} \lambda_{n}^{2}+\sqrt{\left.c^{4} \lambda_{n}^{4}+4 p^{2} \lambda_{n}^{4} M^{4}\right)}\right) / 2} \cdot \lambda_{n}$ is the largest eigenvalue of matrix $L . M=\max \left(\left|\mu_{i}\right|\right)$.

From the above analysis, we have $\tau^{*}<\tilde{\tau}$. When $0<$ $\tau^{*}<\tilde{\tau}$, (19) no longer holds, so there is no nonzero imaginary eigenvalue. When $\tau^{*}=0$, from Lemma 5, we know that only when $\delta(t)=\varepsilon\left[\mathbf{1}_{n}^{T}, 0\right]^{T}$, does (18) have a zero root. In other words, only if $\bar{x}(t)=\varepsilon \mathbf{1}_{n}, \bar{\Theta}(t)=0$, which, according to Definition 3, means that the consensus has been achieved, does the system have a zero eigenvalue. In other situations, the eigenvalues of the system all have negative real parts. So we can come to the conclusion that the consensus of the system can be achieved if the largest time delay satisfies (25). The proof of Theorem 8 is completed.

Remark 9. The given result in Theorem 8 for the upper bound is a not a conservative one. From the proof of Theorem 8 , we know that $\tau^{*}$ has been amplified for several times during the derivation. The least upper bound of the time delay is smaller than the given result, which means that the proposed adaptive control method may not be feasible even when the necessary condition is satisfied.

Theorem 8 is a necessary condition of the maximum time delay $\tau^{*}$ for the system stability. The following theorem gives an explicit result of $\tau^{*}$.

Theorem 10. The consensus for multiagent systems with nonlinear dynamics and time delays using adaptive protocols (7) and (8) can be achieved if the maximum time delay $\tau^{*}$ satisfies

$$
\tau^{*}=\min _{1 \leq i \leq 3 n-2}\left(\min _{1 \leq k \leq n-1} \frac{\sigma_{k}^{i}}{\omega_{k}^{i}}\right)
$$

for some $\omega_{k}^{i} \in(0,+\infty), \sigma_{k}^{i} \in[0,2 \pi)$, where $\sigma_{k}^{i}$ satisfies $e^{-j \sigma_{k}^{i}}=$ $\lambda_{i}\left(G\left(j \omega_{k}^{i}\right), H\right) . \lambda_{i}(G(s), H)$ is the generalized eigenvalue of the matrix pair $(G(s), H)$, and $G(s)=\left(\begin{array}{cc}0 & -I \\ s I-A & -B\end{array}\right)$ and $H=\left(\begin{array}{cc}-I & 0 \\ 0 & C\end{array}\right)$, in which matrices $A, B$, and $C$ are defined in (10).

Proof. From (13), using the Schur complement lemma, we have

$$
\begin{aligned}
\operatorname{det} & \left(s I_{2 n}-A-B e^{-\tau s}-C e^{-2 \tau s}\right) \\
= & \operatorname{det}\left(e^{-\tau s} I_{2 n}\right) \operatorname{det}\left(-C e^{-\tau s}-B+s I_{2 n}-A\right) \\
& =\left|\begin{array}{cc}
I_{2 n} e^{-\tau s} & -I \\
s I_{2 n}-A & -C e^{-\tau s}-B
\end{array}\right| .
\end{aligned}
$$

By extending the order of the matrix, the eigenpolynomial with two delay terms becomes a new eigenpolynomial with a single time delay term as follows:

$$
\operatorname{det}\left(s I_{2 n}-A-B e^{-\tau s}-C e^{-2 \tau s}\right)=\operatorname{det}\left(G(s)-e^{-\tau s} H\right) \text {, }
$$

where $G(s)=\left(\begin{array}{cc}0 & -I \\ s I-A & -B\end{array}\right)$ and $H=\left(\begin{array}{cc}-I & 0 \\ 0 & C\end{array}\right)$.

Whenever $s$ goes across the imaginary axis, there exits $s=j \omega$ so that $e^{-j \omega}$ is a generalized eigenvalue of matrix pair $(G(s), H)$. There are at most $n-1$ frequency $\omega_{k}^{i}$ satisfying $\left\|\lambda_{i}\left(G\left(j \omega_{k}^{i}\right), H\right)\right\|_{2}=\left\|e^{-j \omega_{k}^{i}}\right\|_{2}=1$. Because $\operatorname{rank}(H)=3 n-2$, there are at most $3 n-2$ generalized eigenvalues of $(G(s), H)$ with magnitude 1 . To guarantee the stability of the system, the maximum time delay is obtained as the minimum of all possible $\tau$ for $s$ to cross the imaginary axis. So (26) holds. The proof of Theorem 10 is completed. 


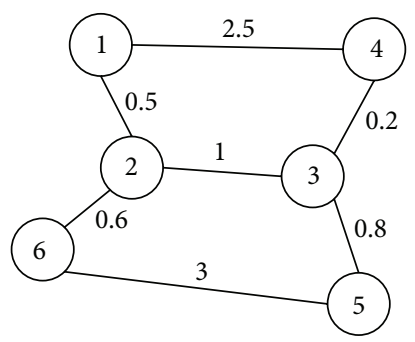

FIGURE 1: Communication topology of the multiagent systems.

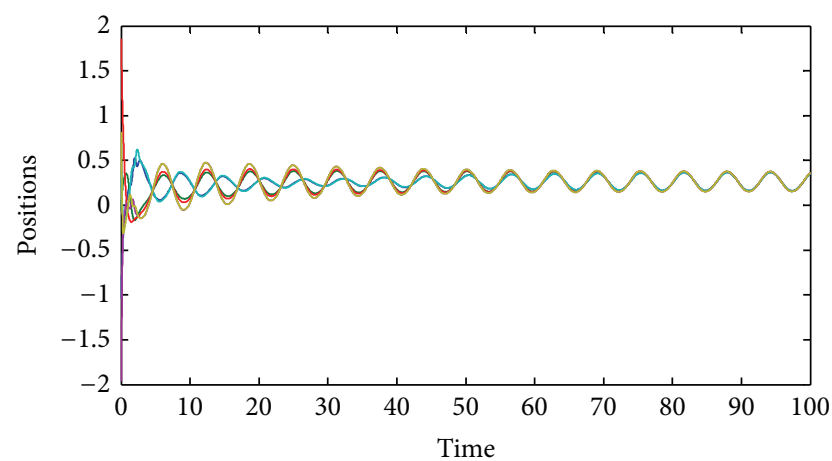

FIGURE 2: The trajectory of agents position states.

\section{A Simulation Example}

In this section, a simulation example is illustrated to demonstrate the effectiveness of the proposed consensus protocol under the given time delays. The nonlinear dynamics of the agents is described as $f_{i}\left(x_{i}, t\right)=\theta_{i} x_{i} \sin (t)$. The time delay is $\tau=0.05$. The communication topology of the 6-node multiagent system is given in Figure 1.

The parameters in this method are chosen as $c=1>$ $0, p=1>0$. According to Theorem 8 , one may obtain that $\tau^{*}<0.104$ in this example. The time delay satisfies $\tau=0.05<0.104$, which is a necessary condition for the consensus. As we can see in Figure 2, using the proposed twohop relay adaptive protocol, the positions of all the agents are synchronized to a common trajectory. Figure 3 shows that the estimation parameter errors of all agents converge to zero. The simulation verifies the theory results that not only the system achieves a consensus but also the parameter errors reach zero under certain time delay situation. It is also illustrated that the convergence cannot be reached when $\tau=0.08$. The reason is, as is implied in Remark 9, that what Theorem 8 presents is a necessary condition, not a sufficient one, and thus it does not guarantee a consensus even when the condition is satisfied.

\section{Conclusions}

This paper proposed a two-hop adaptive control method for consensus of multiagent systems with nonlinear dynamics and time delays. By involving the estimation parameters as

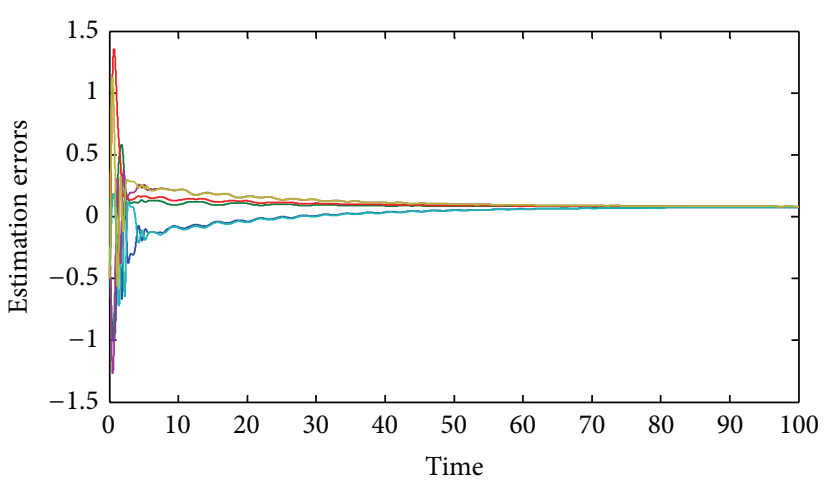

FIGURE 3: The trajectory of estimation parameters.

system states, we rewrite the system model in an extendedorder manner. The maximum tolerated time delay for system consensus is analyzed. A necessary time delay condition and the explicit result are given using a frequency domain approach. Both the agent states errors and the estimation parameter errors can converge to zero. A simulation example verified the theoretical results. Consensus for system with nonlinear dynamics and time delays is a challenging yet meaningful work, so future work will focus on a more conservative upper bound of the maximum time delay to guarantee the nonlinear consensus.

\section{Conflict of Interests}

The authors declare that there is no conflict of interests regarding the publication of this paper.

\section{Acknowledgments}

This work is supported by the National Basic Research Program of China (973 Program no. 2012CB215200), the National Natural Science Foundation of China (no. 51205046), and Fundamental Research Funds for the Central Universities (no. ZYGX2012J093).

\section{References}

[1] R. Olfati-Saber and R. M. Murray, "Consensus problems in networks of agents with switching topology and time-delays," IEEE Transactions on Automatic Control, vol. 49, no. 9, pp. 15201533, 2004.

[2] V. D. Blondel, J. M. Hendrickx, A. Olshevsky, and J. N. Tsitsiklis, "Convergence in multiagent coordination, consensus, and flocking," in Proceedings of the 44th IEEE Conference on Decision and Control, and the European Control Conference (CDC-ECC '05), pp. 2996-3000, Seville, Spain, December 2005.

[3] R. H. Lasseter, "MicroGrids," in Proceedings of the IEEE Power Engineering Society Winter Meeting, pp. 305-308, New York, NY, USA, January 2002.

[4] W. Ren and E. Atkins, "Second-order consensus protocols in multiple vehicle systems with local interactions," in Proceedings 
of the AIAA Guidance, Navigation, and Control Conference, pp. 3689-3701, San Francisco, Calif, USA, August 2005.

[5] W. Yu, W. X. Zheng, G. Chen, W. Ren, and J. Cao, "Second-order consensus in multi-agent dynamical systems with sampled position data," Automatica, vol. 47, no. 7, pp. 1496-1503, 2011.

[6] H. Yang, Z. Zhang, and S. Zhang, "Consensus of second-order multi-agent systems with exogenous disturbances," International Journal of Robust and Nonlinear Control, vol. 21, no. 9, pp. 945-956, 2011.

[7] W. Yu, G. Chen, and M. Cao, "Some necessary and sufficient conditions for second-order consensus in multi-agent dynamical systems," Automatica, vol. 46, no. 6, pp. 1089-1095, 2010.

[8] P. Lin, M. Dai, and Y. Song, "Consensus stability of a class of second-order multi-agent systems with nonuniform timedelays," Journal of the Franklin Institute, vol. 351, no. 3, pp. 15711576, 2014.

[9] Y.-P. Tian and C.-L. Liu, "Consensus of multi-agent systems with diverse input and communication delays," IEEE Transactions on Automatic Control, vol. 53, no. 9, pp. 2122-2128, 2008.

[10] P. Lin and Y. Jia, "Consensus of second-order discrete-time multi-agent systems with nonuniform time-delays and dynamically changing topologies," Automatica, vol. 45, no. 9, pp. 21542158, 2009.

[11] S. Yin, G. Wang, and H. R. Karimi, "Data-driven design of robust fault detection system for wind turbines," Mechatronics, 2013.

[12] S. Yin, S. Ding, A. Haghani, and H. Hao, "Data-driven monitoring for stochastic systems and its application on batch process," International Journal of Systems Science, vol. 44, no. 7, pp. 13661376, 2013.

[13] X. Feng and W. Long, "Asynchronous consensus in continuoustime multi-agent systems with switching topology and timevarying delays," IEEE Transactions on Automatic Control, vol. 53, no. 8, pp. 1804-1816, 2008.

[14] E. Nuño, R. Ortega, L. Basañez, and D. Hill, "Synchronization of networks of nonidentical Euler-Lagrange systems with uncertain parameters and communication delays," IEEE Transactions on Automatic Control, vol. 56, no. 4, pp. 935-941, 2011.

[15] A. Bidram, A. Davoudi, F. L. Lewis, and J. M. Guerrero, "Distributed cooperative secondary control of microgrids using feedback linearization," IEEE Transactions on Power Systems, vol. 28, no. 3, pp. 3462-3470, 2013.

[16] Q. Zhihua, W. Jing, and R. A. Hull, "Cooperative control of dynamical systems with application to autonomous vehicles," IEEE Transactions on Automatic Control, vol. 53, no. 4, pp. 894911, 2008.

[17] H. Li, X. Liao, T. Huang, Y. Wang, Q. Han, and T. Dong, "Algebraic criteria for second-order global consensus in multiagent networks with intrinsic nonlinear dynamics and directed topologies," Information Sciences, vol. 259, pp. 25-35, 2014.

[18] Y. Qian, X. Wu, J. Lü, and J.-A. Lu, "Second-order consensus of multi-agent systems with nonlinear dynamics via impulsive control," Neurocomputing, vol. 125, pp. 142-147, 2014.

[19] H. Yu and X. Xia, "Adaptive consensus of multi-agents in networks with jointly connected topologies," Automatica, vol. 48, no. 8, pp. 1783-1790, 2012.

[20] S. Yin, H. Luo, and S. Ding, "Real-time implementation of faulttolerant control systems with performance optimization," IEEE Transactions on Industrial Electronics, vol. 61, no. 5, pp. 24022411, 2014.
[21] S. Yin, S. X. Ding, A. Haghani, H. Hao, and P. Zhang, "A comparison study of basic datadriven fault diagnosis and process monitoring methods on the benchmark Tennessee Eastman process," Journal of Process Control, vol. 22, no. 9, pp. 1567-1581, 2012.

[22] W. Yu, G. Chen, M. Cao, and J. Kurths, "Second-order consensus for multiagent systems with directed topologies and nonlinear dynamics," IEEE Transactions on Systems, Man, and Cybernetics B, vol. 40, no. 3, pp. 881-891, 2010.

[23] G. H. Wen, Z. S. Duan, W. W. Yu, and G. R. Chen, "Consensus of second-order multi-agent systems with delayed nonlinear dynamics and intermittent communications," International Journal of Control, vol. 86, no. 2, pp. 322-331, 2013.

[24] C. W. Wu and L. O. Chua, "Synchronization in an array of linearly coupled dynamical systems," IEEE Transactions on Circuits and Systems I: Fundamental Theory and Applications, vol. 42, no. 8, pp. 430-447, 1995.

[25] J. Zhipu and R. M. Murray, "Multi-hop relay protocols for fast consensus seeking," in Proceedings of the 45th IEEE Conference on Decision and Control, pp. 1001-1006, San Diego, Calif, USA, December 2006. 


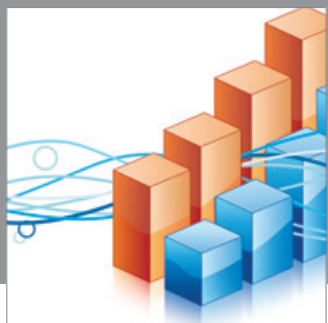

Advances in

Operations Research

mansans

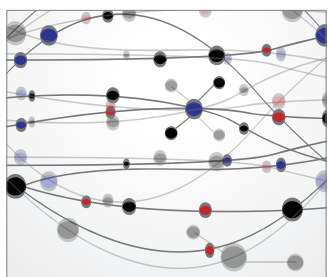

The Scientific World Journal
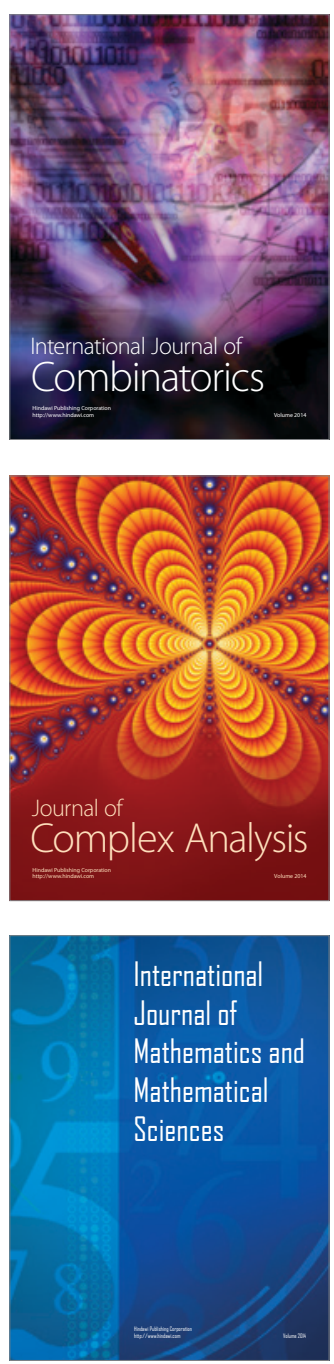
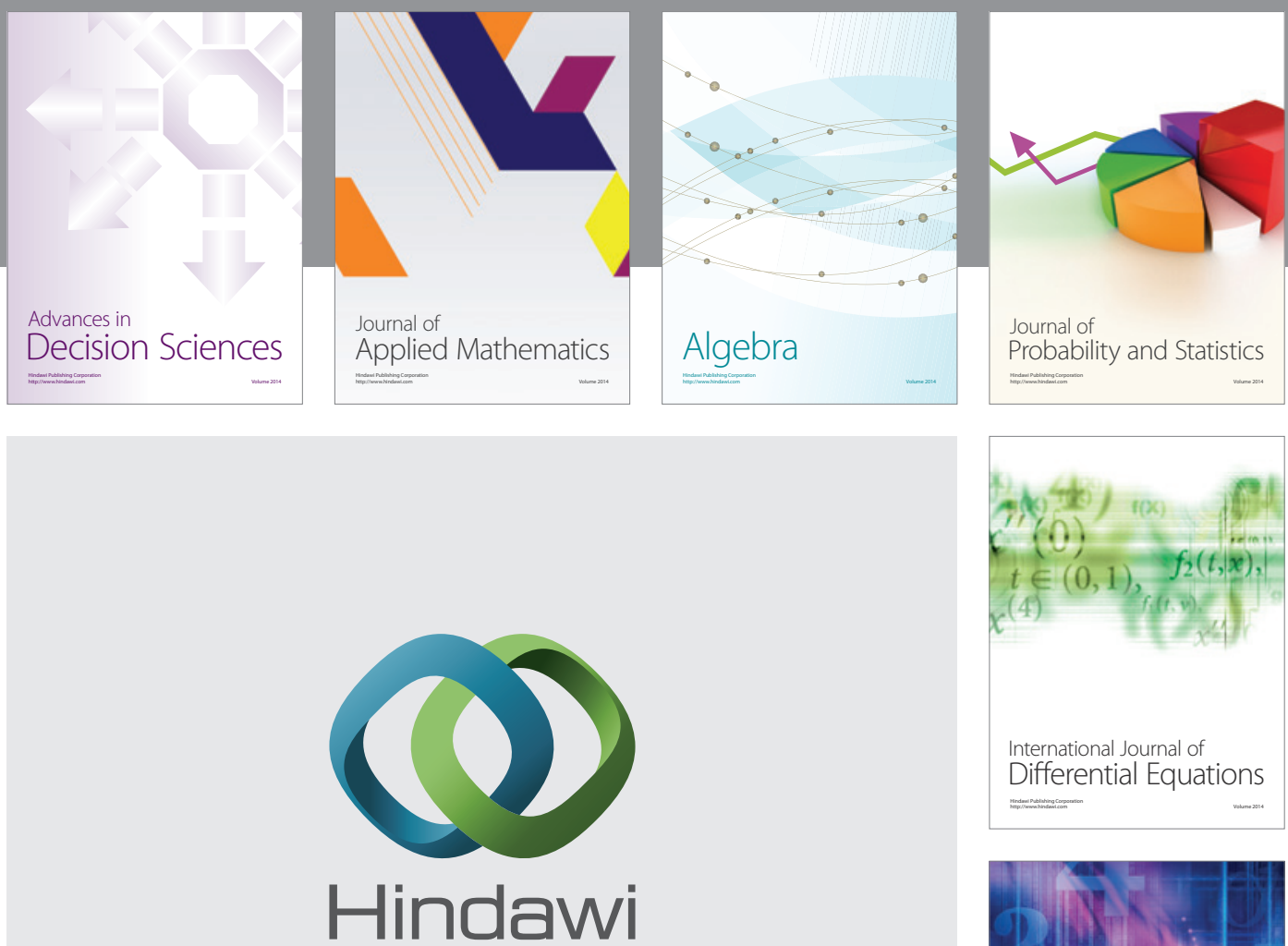

Submit your manuscripts at http://www.hindawi.com
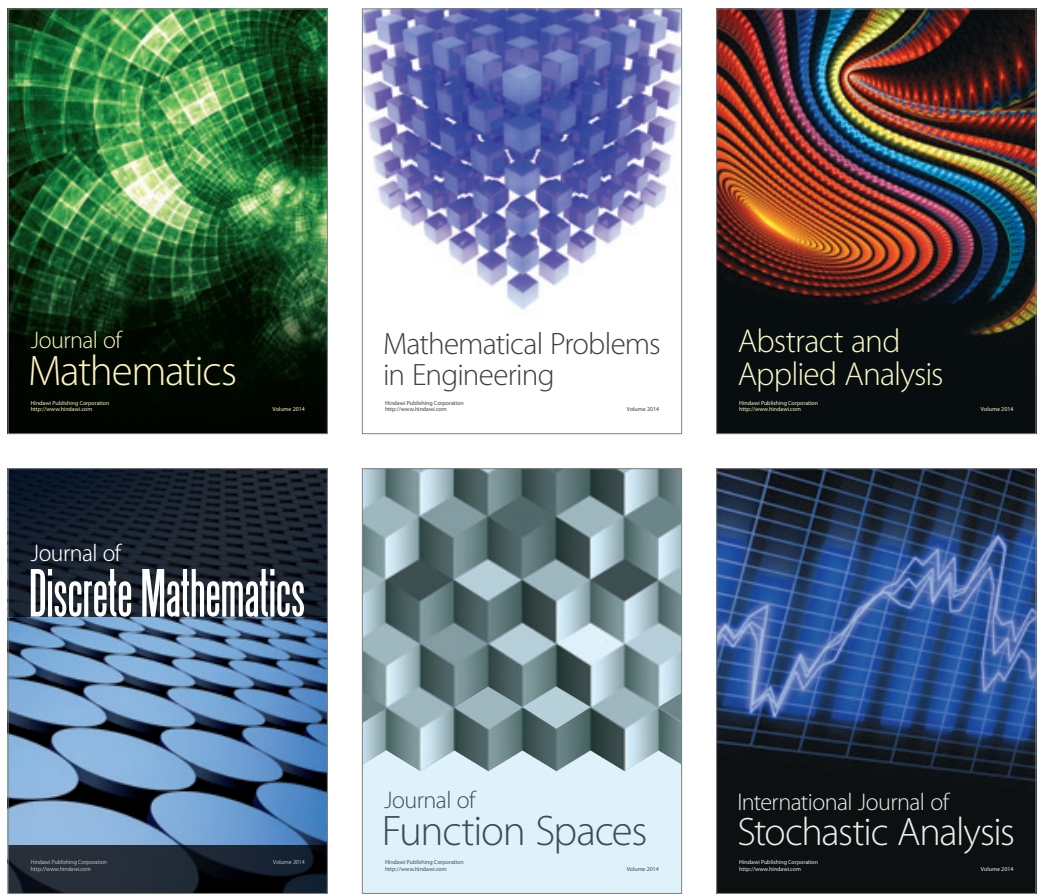

Journal of

Function Spaces

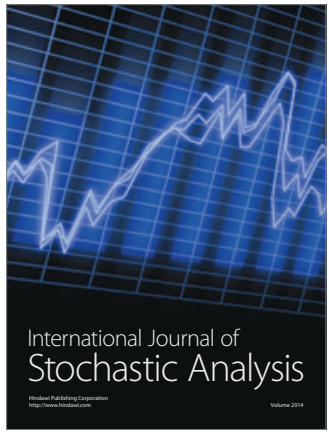

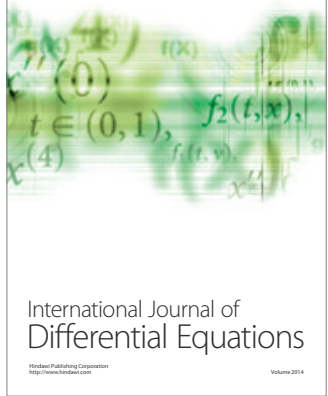
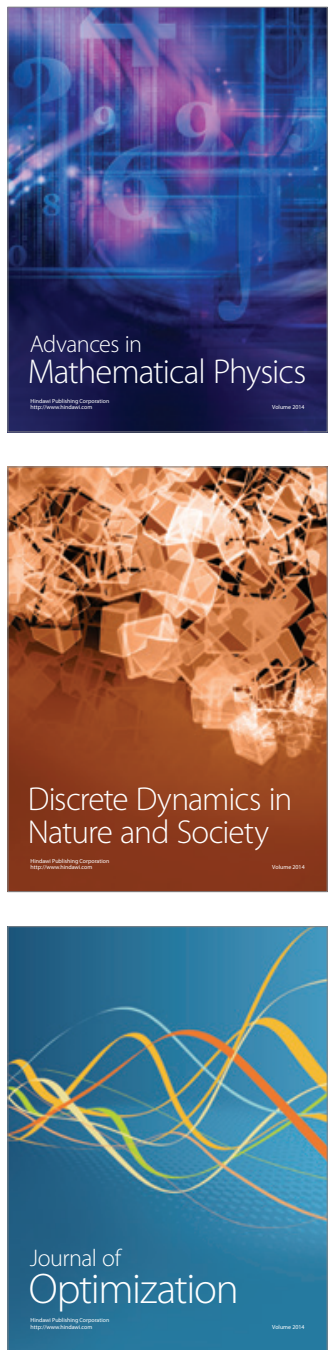